\title{
An Adaptive Interface Design (AID) for enhanced computer accessibility and rehabilitation
}

\author{
Yingzi Lin*, Jeffrey Breugelmans, Maura Iversen, David Schmidt \\ Mechanical and Industrial Engineering Department, College of Engineering \\ Physical Therapy Department, Bouvé College of Health Sciences \\ Northeastern University, Boston, MA USA \\ *Corresponding Author: yi.lin@neu.edu
}

\begin{abstract}
This study was aimed at the development of a nonintrusive, biosensor based, Adaptive Interface Design (AID) that utilizes its users' physical abilities (rather than focusing on their disabilities), while simultaneously honoring their integrity and needs. The target users for this study were patients with arthritis in the hand. The AID comprises of eye tracking and data glove technologies, the latter of which will be used to measure the user's physical ability as it translates hand gestures into computer controls. By developing accessible computer interfaces and exploring their use for telerehabilitation purposes, we want to ultimately impact the inclusion and health outcomes of those who experience disability on a daily basis.
\end{abstract}

Index Terms - Adaptive Interface Design, Computer Accessibility, Rehabilitation

\section{INTRODUCTION}

$\mathrm{C}$ ontemporary paradigms for non-intrusive biosensors consist of wearable and non-contact sensors. Wearable sensors are made as articles that are worn by people in their daily functioning (e.g., watches used for heart rate monitoring [1]) and can be used to acquire a number of physiological signals such as heart rate, skin conductance, etc. [2]. When

This paper was submitted to the IEE Journal on Monday, May $17^{\text {th }} 2016$. This work was supported under NSF Grant \# 0954579.

Y. Lin is with the Department of Mechanical and Industrial Engineering, Northeastern University, Boston, MA 02115 USA (email: yilin@ @ coe.neu.edu) J. Breugelmans and D. Schmidt are in the Department of Mechanical and Industrial Engineering, Northeastern University, Boston, MA 02115 USA, and has since graduated (email: j.breugelmans@husky.neu.edu).

M. Iversen is with the Department of Physical Therapy, Northeastern University, Boston, MA, 02115 (email: m.iversen@neu.edu).

D. Schmidt is a PhD Candidate in the Department of Bioengineering, Northeastern University, Boston, MA 02115 USA (email: schmidt.d@ husky.neu.edu). using wearable sensors, it is important to consider their degree of intrusiveness as they form an additional layer between the human-machine interactions [3]. Non-contact sensors include vision-based sensors such as the tracking of body movements through computer vision [4-9], visual attention tracking through eye-gaze monitoring [10-15] and video/camera systems for facial expressions [16]. For those applications that require contact-based sensing which is ubiquitous in humanmachine interaction [17], Lin developed a new paradigm for biosensors called the natural-contact biosensor [2]. The basic idea of the natural-contact sensor is to build unobtrusive transducers into the surfaces where natural physical contact between the user and the machine occurs. The follow overview discusses some developments in the field of biosensing in relation to computer control, and how these developments might be applied to the field of rehabilitation sciences.

\section{INNOVATIONS IN HUMAN-TECHNOLOGY INTERACTION}

Tracking eye and body movements. Although the field of neuroscience has produced a lot of interesting research related to Brain-Computer Interaction (BCI), this study has been limited in focus to eye and body movement tracking in order to best target those users with physical disabilities, in particular arthritis. Eye tracking has been a topic of research since the 1980's [18]. It can be used to evaluate a person's visual attention and cognitive decision-making process [19], or as a means to facilitate computer input. One such example application is the use of eye-gaze based typing as described by 
[20]. Similarly, eye tracking technology has been used to replace mouse input in video games [21,22]. In an alternative approach, [6] applied a camera to register body movements and translate these into mouse cursor displacements. Other researchers have proposed the use of hand gestures in order to facilitate human-computer interaction [23], which can be achieved either through the use of computer vision [7-9,24$28]$, or by means of glove-based input $[29,30]$. These suggestions found support through the development of gloves that use infrared light to manipulate virtual objects [e.g., 4,31 ], or accelerometers to accurately measure hand movements [e.g., 32]. The drawback of many of these examples, however, is that they have not been designed for people with disabilities, and may therefore offer only limited support.

Perhaps the most studied data glove for rehabilitation is the Rutgers Master Glove II, which has been applied to patients with various physical health conditions, such as stroke, carpal tunnel syndrome [33]. This data glove would be taped to the user's hand to accurately determine the flexion of each digit. Although this technology may have yield accurate measurements, we considered the system too obtrusive and inaccessible, since it requires the help of another individual to put it on.

Full Body Control. Some of the previously described concepts have been commercialized on a large scale by the recent shift towards full-body-movement based video game control, which was first introduced to the general public in 2006 by the Nintendo Wii, and followed quickly in the form of the PlayStation Move, and Microsoft's Xbox Kinect. The main advance compared to contemporary gaming systems is the integration of computer vision to track player movements. As a result, these motions can be directly translated into video-game controls (e.g., punching, steering).

Although these technologies convey a great potential for increasing the amount of fun and physical engagement while playing video games, they provide limited opportunities to assist disabled users. The main reason is that these systems tend to share a prominent reliance on the user's freedom of movement. Systems that rely on computer vision also pose limitations on the space the user can occupy, i.e. the space or body position wherein movements can be accurately registered. However, this has not discouraged researchers from exploring the possibilities of implementing these devices as a means of low-cost rehabilitation [34-42]. What prevents these computer-vision based systems from being used in the proposed application (targeting patients who experience computer accessibility issues due to arthritis in the hand), is the fact that they have clear limitations when it comes to measuring certain muscular performances, for example grip strength, which is a vital component in physical therapy exercises for patients with a rheumatoid hand [43].

Rehabilitation Science. Physical therapists agree that motivation is a key aspect in a successful rehabilitation process $[41,44,45]$. The field of psychology has defined two types of motivation: intrinsic and extrinsic [46]. Intrinsic motivation means that a person performs a task for the enjoyment of the task itself, whereas extrinsic motivation means that a person does a task because of a separate outcome or goal. A combination of both types of motivation is preferred for rehabilitation. Ideally, the experience should be aimed at the end goal of physical recovery, whilst being enjoyable in itself to yield ultimate exercise compliance. Another major factor in a patient's motivation to adhere to physical therapy process is the amount of contact between patient and physician [47]. In order words, patients tend to exercise more if they communicate with their physician more frequently. This is an important drive behind the field of telerehabilitation. In this respect, motivation may be positively affected by sharing the user's performance with a remote physician, allowing him/her to keep close track of the patients' current abilities and overall rehabilitation progress $[48,49]$. When applied effectively, telerehabilitation may even lead to a reduction in the frequency of clinical visits, as well as the time, effort, and costs involved. Telerehabilitation should only be applied to enrich the process, however, not as a means to replace physical care entirely [26'].

Although the application of virtual immersion to rehabilitation sciences can be challenging, it also offers a wide range of potential benefits [50]. Virtual games that are aimed at enhancing physical exercise have been dubbed 'Exergames'

[51]. Research has shown that virtual immersion can be used to reduce stress and can expedite recovery [52-54], and that exergaming can lead to enhanced clinical outcomes by promoting a higher quality of therapeutic support and enhancing exercise adherence among patients with insufficient motivation [55,56]. Furthermore, physical therapy patients often prefer virtual reality exercises to their common exercise routines [57].

\section{PRoposed SOlution}

The Adaptive Interface Design (AID). This paper discusses the development of a computer interface that is aimed at assisting patients with various conditions of arthritis in the hand. By developing a data glove that can measure both finger movements and grip strength $[12,14]$, this wearable sensor can be used to continuously and unobtrusively track the hand movements of the user. It effectively turns the hand into an input device while granting users the freedom to assume whatever hand position they find most comfortable, as it eliminates the need for users to place their hands on a 
controller (e.g., keyboard) or within the limited view of motion capturing cameras. Through the addition of an eye tracking device the user is capable of exploring a virtual environment that has been specially designed for this application. The alternative control method consists of automated camera movements based on the user's eye-gaze, and the use of hand gestures for virtual interactions. When deciding which hand movements to incorporate, a preference goes towards more rapid and course gestures over complex and precise gestures, but this does limit the number of available actions that can be incorporated [58]. The decision was made to include the movements of grasp and release, pronation, and supination, which are all necessary to perform many activities of daily living [31].

Ideally, any system should work according to the "plug-andplay" principle [59], allowing any user to achieve a successful experience upon his/her first encounter with it. It is expected that by modeling the virtual interactions closely to the natural hand movement used in daily practice, the system's operations should be easy to adopt. Among other factors, the system will be tested in regard to 'learnability', in order to assess how challenging it is to first-time users.

Relevant Works. Similar systems comprising of eye tracker and data glove components have been applied to in the visualization of 3D images for physicians and other professionals in the biomedical field $[29,60,61]$. The work by Krabichler et al. can be seen as a pioneering example of the possibilities at hand. However, the main distinctions between that study and ours lie in our choice of users with disability, the different application of human-computer interaction, and the alternative approach in regards to system operation.

\section{RESEARCH OBJECTIVES}

\section{Objective 1: To develop a multi-sensor technology for} acquiring physical operation cues non-intrusively and in real-time. Our plan is to replace the conventional computer interface devices by a more enabling and non-intrusive alternative. This will be achieved by utilizing an eye tracking device to establish a non-contact method for on-screen navigation, and a wearable sensor for the tracking of hand gestures.

Expected Outcome: Through the incorporation of biosensor technologies, the system will enable non-intrusive and realtime data-acquisition.

\section{Objective 2: To develop methods and algorithms for translating the physical interaction cues into real-time virtual controls. This research was focused on two cues: hand movements and visual attention, which are monitored using an eye tracking device as well as flex and pressure sensors embedded in a data glove. These inputs are fused by}

the Unity game environment, and translated into virtual interactions.

Expected outcome: By linking physical behaviors directly to computer controls, the system effectively eliminates the user's dependency on additional input devices that may be more cumbersome to operate. We expect the resulting interaction to be accessible, engaging, and easy to understand.

\section{Objective 3: To investigate the physical control} implications on the user's performance. During rehabilitation practices it is important that the physical ability of the patient is closely monitored. Maintaining an accurate understanding of the user's physical ability will enable the system to: (1) generate better predictions of what physical controls the user might exercise at the moment, and (2) adapt and automatically calibrate to different individuals at any time.

Expected Outcome: By tracking the user's performance and adopting the control parameters continuously, we aim to make the system "grow" along with the user's physical progress; ensuring that the interface will always reflect the user's current ability and offer an appropriate challenge level. In the scope of this paper, we will verify the adaptive properties of the interface. A follow up study should include clinical trials that to evaluate a user's engagement and physical progress throughout prolonged use of the system.

Prospective Outcome: To develop the Adaptive Interface Design (AID) for improved computer accessibility, with a potential application in rehabilitative practices. Because of the relevance of computer accessibility in our increasingly digital society and the predicted increase of users experiencing physical disability, we believe the findings of this work can have a significant contribution to the quality our target users' lives and their rehabilitation practices.

\section{System DEVELOPMENT}

The proposed system consists of two major hardware components, a virtual environment test-bed, and a software environment linking everything together. First there is the eye tracking system that replaces the classical mouse interface for on-screen pointing. Next, the user will interact with the computer through a data glove, which is designed to replace the conventional keyboard input for in-game navigation and interaction. These two inputs are combined to control the virtual interaction in the video game.

System Setup. The system setup includes an eye tracker (placed underneath a 22" LCD monitor) and a data glove, which is worn on the right hand and connected to the computer using a National Instruments Data Acquisition unit (NI-DAQ). A graphical representation of this setup can be found in Figure 1. 


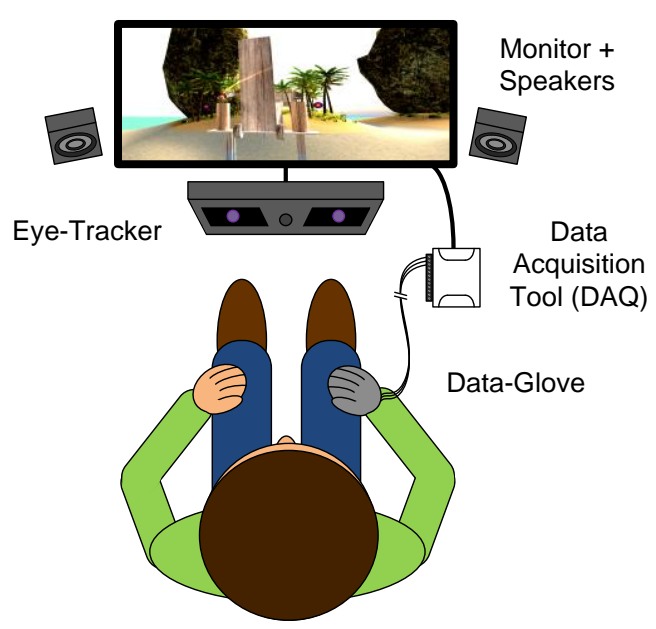

Figure 1: Overview of the Adaptive Interface Design (AID).

\section{COMPONENT 1: EYE TRACKER}

Functionality. A Tobii $x-50$ eye tracker was used in this study. This eye tracker uses infrared LEDs to project infrared light towards the user's eyes. This light is reflected by the user's retinas and picked up by the infrared camera embedded in the system (located in the middle front of the device). Since the eye tracker is placed under the LCD screen, it poses no obstruction to participant's view. After calibration, the system is able to accurately determine the virtual coordinates $(\mathrm{x}, \mathrm{y})$ of the left and right eye fixations. After locating the user's eyefixation on the screen, this information is further processed and utilized as a control input.

Translation into Virtual Controls. The virtual environment was created in Unity [62]. The program contains a camera script that monitors the current eye-gaze position ( $\mathrm{x}$ - and $\mathrm{y}$ coordinates) and determines its offset from the center of the screen. It works in such a way that off-center eye-fixations are followed by a camera rotation in the corresponding direction. Because the speed of the rotation is proportional to the offset, camera movements have a natural dampening effect. Small offsets lead to slow movements, and large offsets result in quicker rotations. As the object of interest gets drawn closer to the center of the screen, the rotation speed automatically slows down. Since the camera view rotates, rather than jumps, towards the eye-gaze position, its movements are fluent and uninterrupted. Whenever the user is focused on something, the view will remain steadily aimed in that direction (e.g., looking at an object or walking down a path). The maximum speed of the camera rotation is limited to ensure an efficient and comfortable roaming experience.

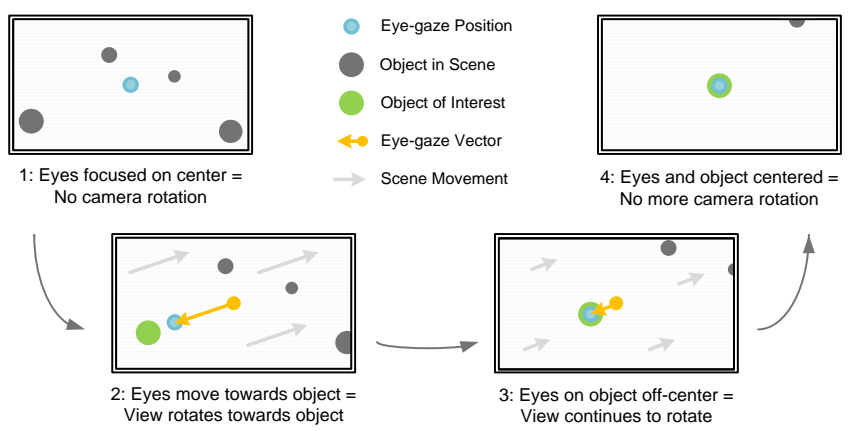

Figure 2: Smooth camera movements based on Eye-Gaze vector; eye gaze location determines direction and speed of camera rotation.

This process is illustrated in Figure 2, wherein the blue dot indicates the current eye-gaze location on the screen and the orange arrow represents the Eye-gaze Vector (offset), which is derived from the current location of the eye-gaze in correspondence to the center of the screen. The following steps describe how an object of interest is focused on:

1. Four Objects are shown within the virtual Scene, but the user's Eye-gaze is positioned at the center of the screen. As a result, the Eye-gaze Vector has no magnitude or direction, and no camera rotation is triggered.

2. The Eye-gaze moves off-center as the user starts to look at the Object of Interest. This assigns both a direction and magnitude to the Eye-Gaze Vector, causing a camera rotation in the corresponding direction. As the view of the scene begins to change, the Object of Interest starts to moves towards the center. The camera rotation is smooth and the speed is higher for eye fixations closer to the edge of the screen.

3. The Eye-Gaze is located directly on the Object of Interest. Because it is not yet centered on the screen, the magnitude of the Eye-gaze Vector remains greater than 0, causing the camera to continue rotating in the direction of the Vector. The closer the object gets to the center of the screen, the slower the corresponding camera rotation.

4. The Scene view gradually centers on the Object of Interest. Once the Object of Interest is in the center of the view, the Eye-Gaze Vector no longer has a direction or magnitude.

Usability. The eye tracking functionality leads to a reduction in required effort, since an eye-fixation automatically leads to camera rotation without the need for an additional manual movement of the mouse in the corresponding direction. It is expected that participants with reduced motor skills in their arms or hands will experience less difficulty in placing the cursor over an object of interest (e.g., a target), because the users' focus in combination with the eye tracking setup will automatically take care of this. 


\section{HERE TO EDIT) <}

\section{COMPONENT 2: DATA Glove}

Functionality. To facilitate the tracking of the user's hand gestures, two types of piezoresistive sensors were embedded into a right-handed glove. The glove's middle finger was fitted with a Flex Sensor. This type of sensor has piezoresistive properties, causing it to have an initial resistance $(\approx 10 \mathrm{k} \Omega)$, which rises exponentially when exposed to increased curvatures. These sensors are compact and fairly cheap to purchase, making them ideal for fabricating a relatively lowcost data glove.

As shown in Figure 3, the amount of flex in the finger was used to dynamically control the virtual walking speed, causing the in-game character to move faster as the sensor's curvature increased. This is not something that conventional keyboard controls can mimic due to their binary properties, and it has the added benefit of enabling the system to accurately track the user's available range of movement in the finger joints (minimum vs. maximum flex).

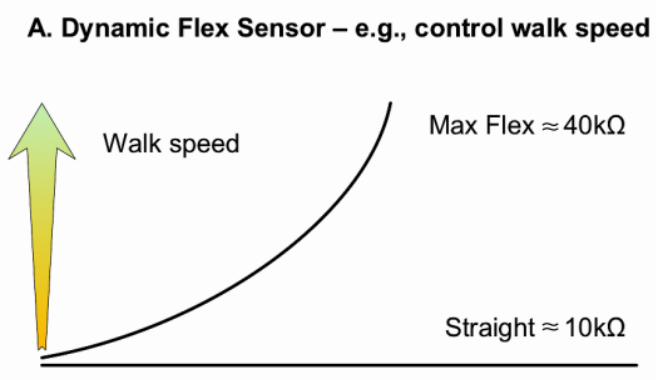

Flex increases $\rightarrow$ Faster walking

Figure 3: Flex sensor application with dynamic signal processing.

Similarly, a Flexiforce resistance sensor was placed on the thumb to function as the 'action trigger'. This Flexiforce sensor also has piezoresistive properties that give it an initially large resistance $(\approx 40 \mathrm{k} \Omega)$. In this case, the sensor's resistance drops as increased pressure is applied, and a dynamic threshold-function was applied to trigger interactions within the virtual environment. The dynamic properties of this threshold will be discussed in the following section. Figure 4 shows a representation of this sensor functionality within the system.

This control triggers different actions depending on the current context of the game. For instance, it triggers jumping in case the user is simply exploring the environment, but allows the player to pick up particular game objects (e.g., a puzzle piece) when facing it. Adding such dynamic properties allows for a reduction in the number of controls needed and makes it easier to keep the system design as simple as possible.

\section{Pressure Sensor Trigger - e.g., object manipulation}

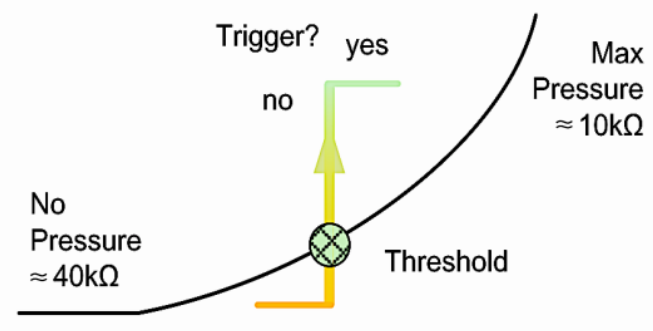

Pressure reaches Threshold $\rightarrow$ Action Trigger

Figure 4: The Flexi Force Sensor functionality within the system.

The data glove was connected to a National Instruments data acquisition system (NI-DAQ USB-6009 14-Bit, 48 KB/s; National Instruments DAQ, 2016), and processed using LabVIEW. Both Transmission Control Protocol (TCP) and User Datagram Protocols (UDP) were considered to provide data communication between LabVIEW and the Unity game engine. One main difference is that TCP includes a verification step that adds reliability, but this comes at the cost of speed. Because the hand gestures needed to be analyzed and communicated in real-time, while occasional packet loss would not be an issue, UDP was deemed more favorable [63]. Automatic Calibration and Adaptability. When observing patients with arthritis an important consideration is that dexterity will vary significantly between subjects and even within the same subject on a day-to-day basis. In order to account for these differences, the system was designed to closely monitor each user's individual ability. To enable more sensible control parameters, it automatically takes into account the minimum and maximum operations a user performs (e.g., hand open/closed as far as comfortably possible). Furthermore, both manipulations were designed to incorporate a threshold value to verify the user's active intent, i.e., to prevent the user from unintentionally triggering an undesired action. In the case of flexing the finger, for instance, the user has to actuate the sensor past $30 \%$ of his or her available range. This threshold value is merely a default value and can be easily changed to any other preferred value (e.g., $20 \%$ to decrease the challenge, or $40 \%$ to increase it). 


\section{HERE TO EDIT) <}

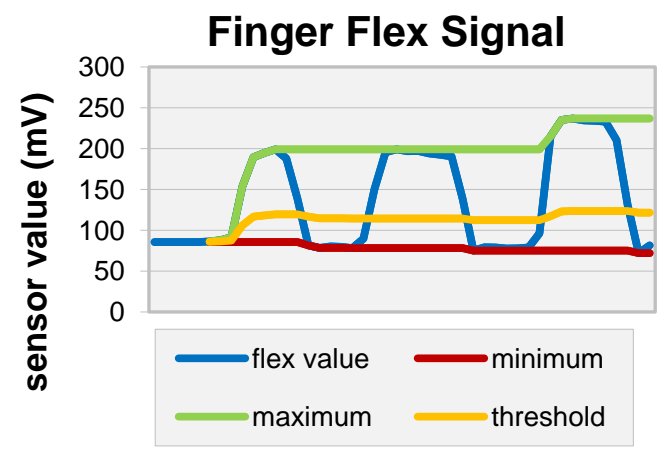

Figure 5: Representation of real-time finger flex data processing, including the instant adaptation of min., max., and threshold values.

Figure 5 shows a representation of the Finger Flex signal processing. As the signal oscillates to reflect the user's hand movements, the software closely monitors the process through the following steps:

1. Observe the Finger Flex signal in real-time (blue).

2. Trace the Maximum (green) and Minimum (red) values to determine the available range of motion.

3. Set the Threshold value (orange) to a preset value (e.g., $30 \%$ ) within this range. Actions are triggered only when the current Flex value exceeds the threshold.

By iterating these steps the system calibrates itself and becomes adaptive by always reflecting the user's current ability. Since the range of mobility may increase over time, it is important to perform these updates. This automatic calibration and adaptation feature offers great potential, especially when using the system as a tool for physical rehabilitation, since it ensures that the user continues to be physically challenged throughout the rehabilitation process.

\section{COMPONENT 3: ViRTUAL ENVIRONMENT}

The final component is the virtual environment itself, which was created using the Unity platform [50]. It lets the player wander around a tropical island in first-person view. This theme provides an excellent starting point for this particular study, because it is very explorative in nature.

Design Considerations. The game was designed for participants to spend most of their time wandering around a nature-rich environment, without being stressed by strictly enforced performance-oriented goals or time constraints. The environment has been enriched with several built features to spike the user's curiosity, and includes some nonthreatening wildlife and a soundtrack of various songbirds and crickets. It is expected that all of these factors have a positive impact on the virtual experience, and thereby increase user engagement, feelings of immersion, and the overall sense of "presence".
Game Objectives. The player's objective is to explore the island and search for a tool that can be used to start a campfire at the end of the journey (to signal for rescue). The player will encounter collectible tokens as well as several mini-games, which were incorporated to make the game more engaging and entertaining. These games were designed to be somewhat challenging for the user, while at the same time demonstrating the versatile capabilities of the alternative interface design. Hence, the way the user interacts with the environment may vary depending on the situation. Figure 6 shows an overview of the island, including the location of the various challenges.

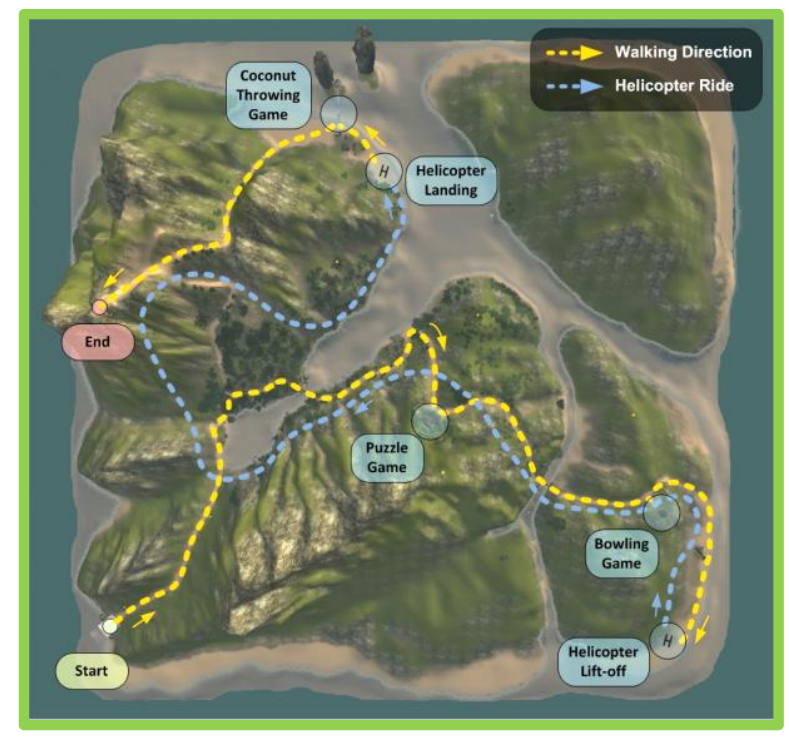

Fig. 6. Island map overview, including the route of the walking path, helicopter ride, and the locations of the mini games.

Start. The player begins his/her journey at the shore on the bottom left side of the map. A brief introduction exercise is given to provide players with some time and practice to familiarize themselves with the controls. Once the player is prepared, he or she will begin to follow a clear path across the island, as indicated by the orange trail on the map. During this first stretch, the player will encounter several collectable tokens that are placed in easy-to-spot locations.

Mini Games. As the player continues to explore the island, he or she will encounter four optional mini games, which include a puzzle, a bowling game, a helicopter ride, and a target practice game. These games were not designed to reflect specific rehabilitative practices, but rather to entice the user to exercise more repeated hand gestures. Whereas flexing of the fingers was mainly linked to the action of walking, the pinching gesture was used as a context sensitive command to pick up, place, roll, and throw objects in order to complete the mini game challenges. When playing the puzzle game, the screen is locked onto the puzzle board and the user moves a cursor on the screen using eye movements. In this scenario, a 


\section{$>$ REPLACE THIS LINE WITH YOUR PAPER IDENTIFICATION NUMBER (DOUBLE-CLICK HERE TO EDIT) <}

pinch gesture is used to pick and place gears into specific slots. The second instance of alternative control occurs during the helicopter ride mini game. In this case, the helicopter follows a pre-set path, but the speed is controlled through hand flex, similar to walking.

End Game. To finish the game, the user walks to the end of the path and uses the matches (won in the puzzle game) to start the campfire using a final pinching gesture.

\section{PRoposed IMPLEMENTATION TO THE FIELD OF TELEREHABILITATION}

Although the scope of this paper covered the development and validation of the proposed interface, we envision a future application in which remote physicians can not only observe, but also modify certain parameters in the interface (see Figure 7). This could be achieved by making adjustments to the sensor processing parameters (e.g., reducing the required physical effort), or by changing some settings in the virtual environment (e.g., enforcing time-constraints, limiting the number of attempts, or modifying other performance oriented factors). These modifications are expected to prolong user engagement by increasing both intrinsic and extrinsic motivation.

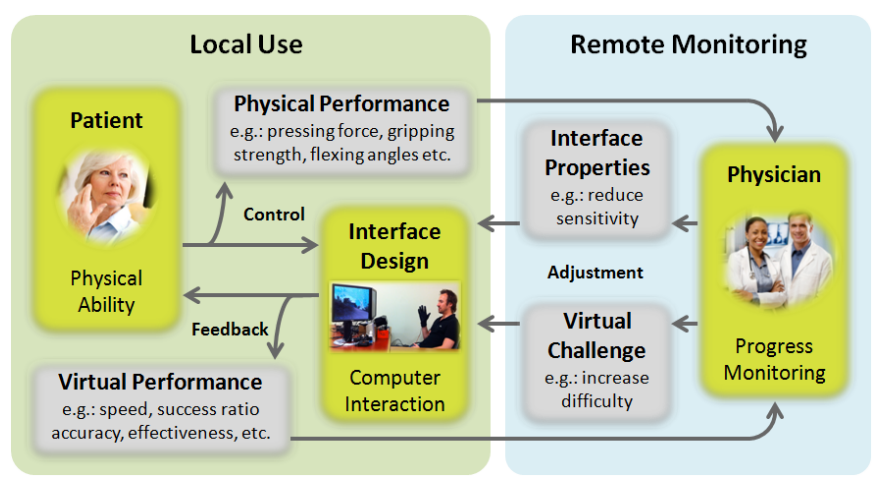

Figure 7: Proposed interplay between the patient, interface, and a remote physician.

Although this feature was not utilized during the test trial described in this paper, Figure 8 provides a representation of the remote data visualization that the system is capable of, in this case using an iPad. This simplistic display provides a real-time link to the data glove signals being processed in LabVIEW. The fader on the left represents the current amount of finger flex exercised by the user (in red; based on the person's range), and the second fader displays the current amount of thumb pressure applied (in green; also based on personal range). The third indicator (in orange) lights up once the user surpasses the thumb pressure threshold and activates the "Pinch" action.

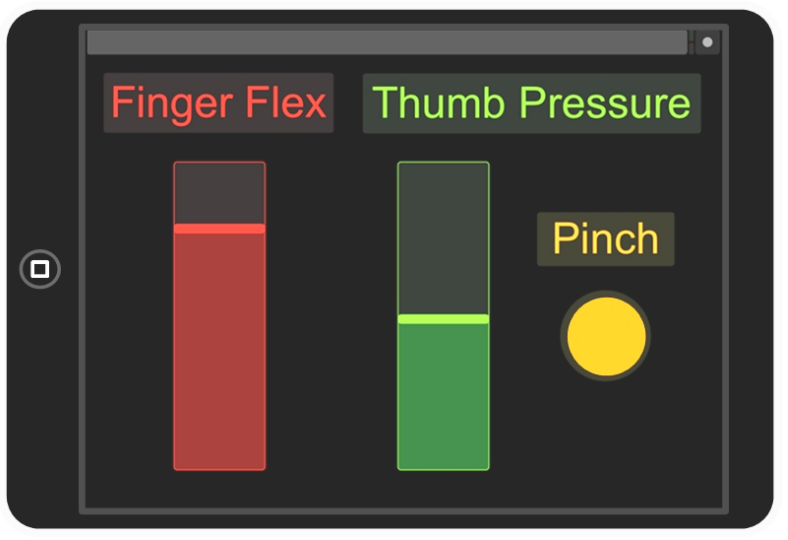

Figure 8: Rendering of a simplistic remote performance monitoring display, using a tablet with UDP connection.

\section{Research Methods}

Subject Recruitment. In an agile approach and in accordance with the principles by [64], the decision was made to test the system frequently with limited numbers of participants per cycle. Earlier trials were conducted with healthy subjects to verify the systems functionality and usability, before involving users with physical disabilities. Several iterations had lead to the current interface design, and this paper describes the results of the final trial using 7 participants with various conditions of arthritis in the hand. These subjects ranged between 20 and 60 years old, and were familiar with conventional computer use. None of them had experienced eye tracking or data glove technologies before.

Apparatus. For an elaborate description of the system component, please refer back to the System Development section of this paper. In short, the AID consists of an eye tracker and data glove component, using a virtual environment as the test bed for user interaction.

Procedure. The decision was made to involve each participant in a single test trial, instead of repeated uses that would enable practice with the system. Since 'Learnability' plays a large factor in the System Usability Scale, it was decided that it would be most interesting to investigate how users would fare during their initial interaction with the system.

At the start of each trial, participants were welcomed to the lab and provided written consent before commencing the study. A brief health assessment was completed, to verify that the user would be able to use the interface without risk of injury. After a verbal introduction to the system and calibration of the eye tracker, each user was asked to put on the glove and explained how to execute the virtual controls (flexing and pinching gesture). Once the users were ready to begin the trial, the virtual environment was loaded, wherein they performed a small introductory task of looking around and grasping objects to familiarize themselves with the 


\section{HERE TO EDIT) <}

controls. When this task was completed, the users were granted access to the full island and encouraged to explore. The trial ended when the user completed the game, or if he or she indicated a desire to stop. No subjects chose to terminate the game before completion.

\section{MEASUREMENT INSTRUMENTS}

Physical Performance. The system was designed to log all finger flex and thumb pressure signals during use. These hand exercises are processed and utilized by the system to enable virtual interactions in real-time. Although not included in the scope of this study, we envision a scenario in which the patient and his/her physician can review these data files to observe changes in ability over time.

Game Experience Questionnaire (GEQ). Once participants completed testing the system, they were asked to evaluate their virtual experience by filling out a modified version of the Game Experience questionnaire (GEQ) [65]. The GEQ has been designed to quantify a subject's objective ratings on the experiential dimensions of perceived enjoyment, flow, immersion, suspense, competence, negative affect, control, and social experience. The social dimension was removed for this study, because it holds no relevance to the current application.

Each of the remaining seven components consists of several statements. The subject's agreement to these statements is measured on a 5-point Likert scale ranging from 0 (strongly disagree) to 5 (strongly agree). A total of 33 items remained on the GEQ that we used, and each component score will be computed as the average value of its sub-items. This questionnaire can provide useful insights on the parts of the experience that perform better than others. Since the AID was being developed to provide an efficient and pleasant computer interaction, it was anticipated that subjects would rate the experience highly on all factors.

System Usability Scale (SUS). After each trial, the participants would also rate the usability of the system they had just used. For the purpose of this study, the decision was made to feature the System Usability Scale (SUS) as developed by [66]. It consists of only 10 questions, but it has proven highly reliable at determining the following aspects: effectiveness, efficiency, and satisfaction. Other surveys were considered [e.g., 67,68], but these tended to be too lengthy or focus on less applicable aspects such as productivity and timemanagement.

\section{OUTCOMES}

It is important to note that the test trials were mainly focused on evaluating the functionality and usability of the developed interface, not their clinical benefits. The AID went through several iterations throughout its development, and these results cover the most recent trial using 7 participants.

Physical Performance Data. Graph 1 on the next page provides a 2-minute sample of the collected data glove signals during a single user's test trial. As the chart indicates, the average baseline for the flex signal (in blue) is situated at around $160 \mathrm{mV}$, signifying the basic position of the opened hand (hand relaxed, not walking). Any time the user would introduce more flex to the finger, the sensor's output signal increased correspondingly. In terms of the virtual interaction, this would result in an increase in forward momentum, provided that the amount of flex reached past the adaptive threshold on the available range (to avoid unintentional movements). When looking at the interval between 60 and 90 seconds, it is clear to see that the user went from standing still $(60 \mathrm{~s}-70 \mathrm{~s})$, to an average walking speed (70s - 80s),

following by the fasted walking speed ( 80 s - 90s) triggered by the maximum amount of flex exerted.

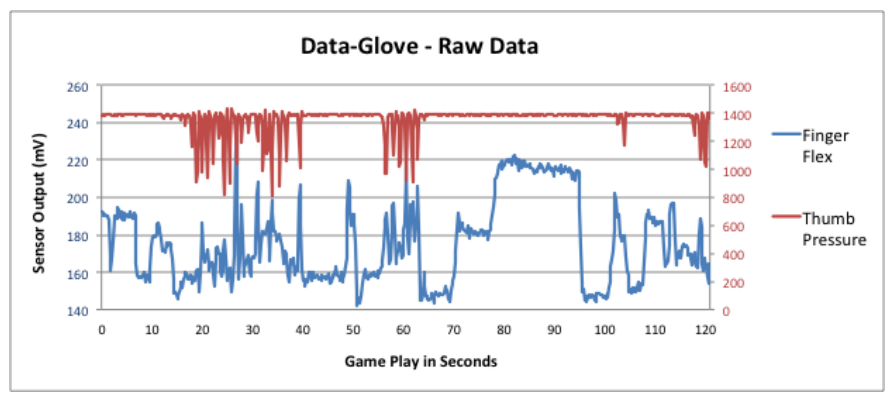

Graph 1: Sample of physical performance data.

The second signal represents the thumb pressure response (in red). Unlike the flex sensor where the hand may always contain some minimal degree of flex, the output of this sensor will have an absolute 0 value when no pressure is being applied. In this case, 0 pressure results in a signal of about $1400 \mathrm{mV}$. In contrast to the flex sensor signal, the output of the pressure sensor drops when activated, as illustrated by the downward spikes in the chart. Each of these spikes represents a virtual interaction, picking up an object for example, given that the threshold function was surpassed.

Game Experience Questionnaire (GEQ) Data. A GEQ was provided after use in order to evaluate the virtual experience. The results are displayed in Graph 2. It can be observed that the experience ratings were generally very positive. 


\section{HERE TO EDIT) <}

Game Experience Questionaire - Outcome

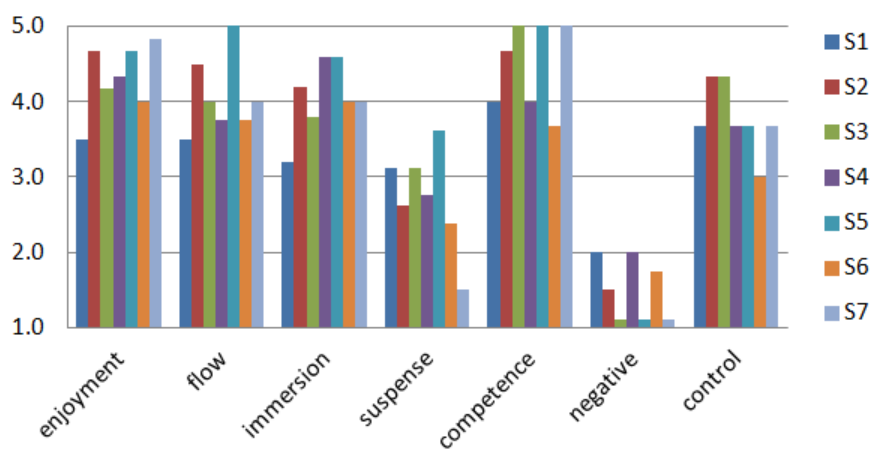

Graph 2: GEQ evaluations after testing the AID.

The high ratings in the enjoyment category and low ratings in the negative categories speak for themselves. The test subjects also indicated a positive level of flow and immersion, meaning that they were very focused on their task and felt immersed in the virtual environment. Another noteworthy finding is that players felt very competent while using the interface and playing the game. One scale to receive lower ratings was the suspense category. This ought to be expected since the goal was to provide a pleasant and stress-free experience. Hence, it appeared that the interface and game succeeded at providing a very positive experience to the users, despite their disability.

System Usability Scale (SUS) Outcomes. For the final assessment, subjects were asked to rate the system based on the 10-item SUS. Generally speaking, a SUS rating above 65 is considered acceptable. Graph 3 displays the outcomes of this evaluation.

\section{System Usability Scale - Outcome}

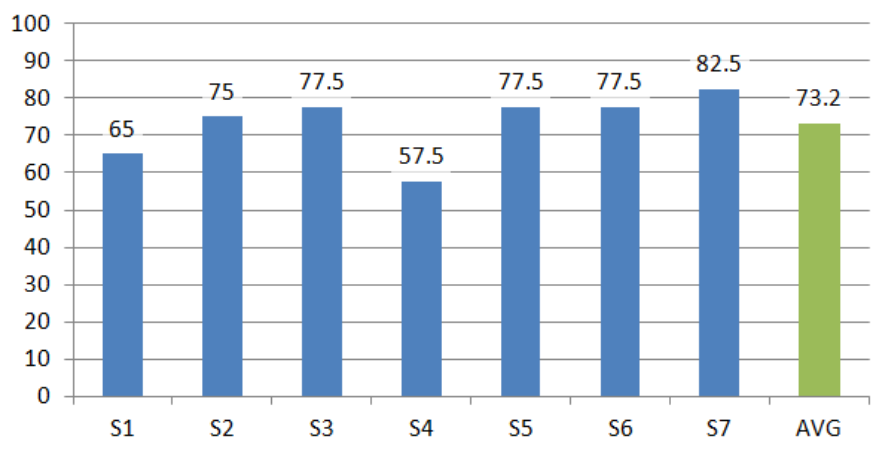

Graph 3: System Usability Scale ratings, including average.

As displayed in this graph, the system was rated at an average score of 73.2. Subject 4 had some difficulty with the eye tracking controls and was the only person to issue a subpar evaluation (57.5). It was observed that this problem resulted from too much head movement while exploring the virtual environment, causing the eye tracker to perform unreliably.
This is something that would be easily remedied once the user is more familiar with the interface.

With possible ratings ranging from 1 to 5, participants were in agreement that they "[...] felt confident while using the system" $(\mathrm{M}=4.1, \mathrm{SD}=0.7)$, and "[...] would like to use the system more frequently" $(\mathrm{M}=4.3, \mathrm{SD}=0.5)$. They also agreed that it was "[...] easy to use" $(\mathrm{M}=4.1, \mathrm{SD}=0.7)$, and that they "[...] would imagine that most people would learn to use this system very quickly" $(\mathrm{M}=4.0, \mathrm{SD}=0.0$; all subjects indicated the same level of agreement). They commonly disagreed with statements that they "[...] needed to learn a lot of things before [they] could get going with the system" $(\mathrm{M}=1.7$, $\mathrm{SD}=0.8)$, and that the system was" [...] unnecessarily complex" $(\mathrm{M}=1.9, \mathrm{SD}=0.7)$, or "[...] awkward to use" $(\mathrm{M}=1.6, \mathrm{SD}=0.8)$.

These results seem to indicate that the subjects enjoyed using the system and that it performed well in terms of Learnability. This is particularly promising when considering the fact that this was merely a first introduction for these users, and that none of them were familiar with eye tracking or data glove technologies. Naturally, there is room for improvement, but these results support the system's feasibility when applied to users with arthritis that have no prior experience with this type of interface.

\section{DisCUSSION AND CONCLUSIONS}

System Development. The Alternative Interface Design (AID) went through several iterations before taking on its final form. The most notable upgrades were applied to the eye tracking algorithm and data glove hardware. During each cycle of the development process the system was tested in order to verify its functioning and usability. This study covers the final test trial involving target users with arthritis.

Performance Data. Apart from serving as an input device, the AID also provides a measure of the user's physical ability. This data can be reviewed by the user, or by a physician in the application of physical rehabilitation. This study was focused on validating the functionality and usability of the AID, but future testing will be needed in order to verify its use in a clinical setting.

Automatic Calibration. As was illustrated in Figure 5, the automatic calibration function in the final version of the system appears to work well. It shows how the observed minimum and maximum are automatically adapted when the user surpasses a previous value, as is the corresponding threshold value. This functionality enables the system parameters to constantly resemble the current user's physical ability. We believe that this feature significantly improves the functionality and usability of the system, since it eliminates the need for a priori calibration or post hoc alterations (e.g., after finding out that the system was too sensitive or not 
sensitive enough). Apart from making the system more accessible for all individuals during initial use, we also foresee that the AID will promote long-term engagement by automatically "growing" along with the user during prolonged and/or recurring use.

Rehabilitation. Having a subject repeatedly interact with the system could provide insights in the physical recovery process. A patient might start off with very limited dexterity, for instance, but consistent use of the AID could reveal a gradual increase in the range of finger flex and amount of grip strength the user can exert. The final version of the AID has demonstrated an ability to communicate the data in real-time. Hence a follow up study could include performance monitoring by a remote physician, who might decide to make changes to the system parameters in real-time (e.g., increasing the difficulty level by adjusting the activation threshold).

User Experience. We applied a modified version of the Game Experience Questionnaire (GEQ) to assess the user's experience in terms of enjoyment, flow, immersion, suspense, competence, negative affect, and control. The experience provided by the final version of the AID proved to be highly immersive and enjoyable, while also granting the players a high sense of competence. The suspense category was the only one in which the final system achieved lower scores on average, but this is to be expected in a game that was designed to be pleasant in nature. The results of this study reveal that the AID was successful at providing a pleasant and immersive experience for users with arthritic conditions in the hand. System Usability. The second post hoc measurement utilized the System Usability Scale (SUS) to analyze the usability of the interface itself. Generally, a SUS score of 65 is considered acceptable, while the final version of the AID received an average score of 73.2. This means the system is regarded in positive light, but that there is also room for improvement. The system scored well in terms of We must keep in mind that the system interactions vary significantly from the standard computer interface that people have grown accustomed to. Hence it should be expected that some adjustment period would be required, before people will be fully comfortable with the alternative control method. This study proved the merit of the AID and serves to justify its further exploration in terms of clinical performance.

\section{ACKNOWLEDGMENT}

We thank the editor and the reviewers for their constructive comments.

This research was supported under NSF grant \# 0954579 awarded to the corresponding author.

\section{REFERENCES}

[1] Picard, R.W. (1997). Affective Computing, Cambridge, Mass.: MIT Press.

[2] Lin, Y. (2011). A natural contact sensor paradigm for non-intrusive and real-time sensing of bio-signals in human-machine interactions. IEEE Sensors Journal, Special Issue on Cognitive Sensor Networks, 11(3):522-529.

[3] Lin, Y. (2008). Towards a new approach for nonintrusive sensing of human bio-signals in human-machine interaction. The 4th International Workshop on Advanced Smart Materials and Smart Structures Technologies (ANCRiSST 2008), Tokyo, Japan.

[4] Micelli, V., Rosiello, F., Aleotti, J., \& Caselli, S. (2009). Design of Gestural Interfaces for Simulated Smart Environments, Adjunct Proceedings of $3 r$ European Conference on Ambient Intelligence (AmI09), pp. 231-234, ISBN 978-3-902737-00-7, Salzburg, Austria, November 2009, M. Tscheligi et al. (Eds.), ICT\&S Center, University of Salzburg.

[5] Placidi, G. (2007). A smart virtual glove for the hand telerehabilitation. Computers in Biology and Medicine, 37:1100-1107.

[6] Betke, M., Gips, J., \& Fleming, P. (2002). The camera mouse: Visual tracking of body features to provide computer access for people with severe disabilities. IEEE Transactions on Neural Systems and Rehabilitation Engineering, 10(1).

[7] Pavlovic, V., Sharma, R., \& Huang, T. (1995). Visual interpretation of hand gestures for human-computer interaction: A review, Technical Report. UIUC-BI-AI-RCV-95-10, University of Central Florida, 1995.

[8] https://www.leapmotion.com/

[9] S. Basso Moro, S. Bisconti, M. Muthalib, M. Spezialetti, S. Cutini, M. Ferrari, G. Placidi and V. Quaresima, "A semi-immersive virtual reality incremental swing balance task activates prefrontal cortex: A functional near-infrared spectroscopy study", NeuroImage, vol. 85, pp. 451-460, 2014.

[10] Lin, Y., Zhang, W. J., Wu, C., Yang, G., \& Dy, J. (2009). A fuzzy logics clustering approach to computing human attention allocation using eyegaze movement cue. International Journal of HumanComputer Studies, 67(5):455-463.

[11] Lin, Y., W.J. Zhang, \& L.G. Watson. (2003). Using eye movement parameters for evaluating human-machine interface frameworks under normal control operation and fault detection situation. International Journal of Human-Computer Studies. 59(6):837-873.

[12] Breugelmans, J., \& Lin, Y. (2011a). Biosensor Based Video Game Control for Physically Disabled Gamers. ASME Dynamic Systems and Control Conference (DSCC). DSC11-6000. Washington, D.C.

[13] Breugelmans, J., \& Lin, Y. (2011b). Incorporating Operator States in Human Assistance Systems Control. International Conference on Transportation Information and Safety (ICTIS). Wuhan, Hubei, China.

[14] Breugelmans, J., Lin, Y., Mourant, R. R., \& Iversen, M. D. (2010). Biosensor-based video game control for physically disables gamers. The 54th Human Factors and Ergonomics Society Annual Meeting. San Francisco, California.

[15] Breugelmans, J., Lin, Y., \& Cai, H. (2009). Incorporating operator states in human assistance systems control. ASME Dynamic Systems and Control Conference (DSCC). Hollywood, CA.

[16] Pantic, M., \& Rothkrantz, L. J. M. (2003). Toward an affect-sensitive multimodal human-computer interaction. Proceedings of the IEEE, 91(9):1370-1390.

[17] Meng, Q., \& Lee, M. H. (2006). Design issues for assistive robotics for the elderly. Advanced Engineering Informatics, 20(2):171-186.

[18] Majaranta, P. \& Räihä, K-J. (2002). Twenty Years of Eye Typing: Systems and Design Issues. Proceedings of ETRA 2002, Eye Tracking Research and Applications, Symposium 2002, New Orleans, LA, ACM Press, March 2002, 15-22. DOI: 10.1145/507072.507076 (ACM). 
[19] Guazzini, A., Yoneki, E., \& Gronchi, G. (2015). Cognitive dissonance and social influence effects on preference judgments: An eye tracking based system for their automatic assessment. International Journal of Human-Computer Studies, vol 73, pp. 12-18.

[20] Frey, L. A., White, K. P. Jr., \& Hutchinson, T. E. (1990). Eye-Gaze Word Processing, IEEE Transactions on Systems, Man, and Cybernetics 20 (4), 944-950.

[21] Smith, J. D., \& Graham, T. C. N. (2006). Use of eye movements for video game control. Proceedings of the ACM conference on Advances in Computer Entertainment Technology, 20-28.

[22] Nacke, L., Stellmach, S., Sasse, D., \& Lindley, C. A. (2009). Gameplay experience in a gaze interaction game. Proceedings of the 5th Conference on Communication by Gaze Interaction - COGAIN 2009: Gaze Interaction For Those Who Want It Most, (Lyngby, Denmark, 2009), The COGAIN Association, 49-54.

[23] Rempel, D., Camilleri, M. J., \& Lee, D. L. (2014). The design of hand gestures for human-computer interaction: Lessons from sign language interpreters. International Journal of Human-Computer Studies, vol. 72, pp. 728-735.

[24] Z. Liu, Y. Zhang, P. Patrick Rau, P. Choe and T. Gulrez, "Leap-Motion Based Online Interactive System for Hand Rehabilitation", CrossCultural Design Applications in Mobile Interaction, Education, Health, Transport and Cultural Heritage, pp. 338-347, 2015.

[25] A. Petracca, M. Carrieri, D. Avola, S. Basso Moro, S. Brigadoi, S. Lancia, M. Spezialetti, M. Ferrari, V. Quaresima and G. Placidi, "A virtual ball task driven by forearm movements for neuro-rehabilitation", 2015 International Conference on Virtual Rehabilitation (ICVR), 2015.

[26] S. Basso Moro, M. Carrieri, D. Avola, S. Brigadoi, S. Lancia, A. Petracca, M. Spezialetti, M. Ferrari, G. Placidi and V. Quaresima, "A novel semi- immersive virtual reality visuo-motor task activates ventrolateral prefrontal cortex: a functional near-infrared spectroscopy study", J. Neural Eng., vol. 13, no. 3, p. 036002, 2016.

[27] D. Avola, M. Spezialetti and G. Placidi, "Design of an efficient framework for fast prototyping of customized human-computer interfaces and virtual environments for rehabilitation", Computer Methods and Programs in Biomedicine, vol. 110, no. 3, pp. 490-502, 2013.

[28] G. Placidi, D. Avola, D. Iacoviello and L. Cinque, "Overall design and implementation of the virtual glove", Computers in Biology and Medicine, vol. 43, no. 11, pp. 1927-1940, 2013.

[29] Zudilova-Seinstra, E. V., de Koning, P. J. H., Suinesiaputra, A., van Schooten, B. W., van der Geest, R. J., Reiber, J. H. C., \& Sloot, P. M. A. (2010). Evaluation of 2D and 3D glove input applied to medical image analysis. International Journal of Human-Computer Studies, vol. 68, pp. 355-369.

[30] Sturman, D. J., \& Zeltzer, D. (1994). "A survey of glove-based input," IEEE Comput. Graph. Applicat. Mag., vol. 14, pp. 30-39.

[31] P. Standen, K. Threapleton, L. Connell, A. Richardson, D. Brown, S. Battersby, C. Sutton and F. Platts, "Patients' Use of a Home-Based Virtual Reality System to Provide Rehabilitation of the Upper Limb Following Stroke", Physical Therapy, vol. 95, no. 3, pp. 350-359, 2014.

[32] Xu, Z., Xiang, C., Wen-hui, W., Ji-hai, Y., Lantz, V., \& Kong-qiao, W. (2009). Hand Gesture Recognition and Virtual Game Control Based on 3D Accelerometer and EMG Sensors. International Conference on Intelligent User Interfaces, 401-406.

[33] Heuser, A., Kourtev, H., Winter, S., Fensterheim, D., Burdea, G., Hentz, V., \& Forducey, P. (2007). Telerehabilitation Using the Rutgers Master II Glove Following Carpal Tunnel Release Surgery: Proof-of-Concept. IEEE Transactions on Neural Systems and Rehabilitation Engineering, 15(1):43-49.

[34] Bo, A. L., Hayashibe, M., \& Poignet, P. (2011). Joint Angle Estimation in Rehabilitation with Inertial Sensors and its Integration with Kinect. Engineering in Medicine and Biology Society, (EMBC), 2011 Annual International Conference of the IEEE. 3479-3483. IEEE.
[35] De Mauro, A. (2011). Virtual Reality Based Rehabilitation and Game Technology. EICS4Med 2011.

[36] Deutsch, J., Borbely, M., Filler, J., Huhn, K., \& Guarrera-Bowlby, P. (2008). Use of a Low-Cost, Commercially Available Gaming Console (Wii) for Rehabilitation of an Adolescent With Cerebral Palsy. Physical Therapy, 88-1196.

[37] Yavuzer, G., Senel, A., Atay, M., \& Stam, H. (2008). Playstation eyetoy games improve upper extremity-related motor functioning in subacute stroke: a randomized controlled clinical trial. Eur J Phys Rehabil Med, 44:237-244.

[38] K. Bakhti, D. Mottet, G. Mélia and I. Laffont, "Low cost objective diagnosis of learned non-use of the paretic arm after a stroke using Kinect technology", Annals of Physical and Rehabilitation Medicine, vol. 58, pp. e11-e12, 2015.

[39] R. Llorens, J. Latorre, E. Noé and E. Keshner, "Posturography using the Wii Balance BoardTM", Gait \& Posture, vol. 43, pp. 228-232, 2016.

[40] G. Palacios-Navarro, I. García-Magariño and P. Ramos-Lorente, "A Kinect-Based System for Lower Limb Rehabilitation in Parkinson's Disease Patients: a Pilot Study", J Med Syst, vol. 39, no. 9, 2015.

[41] M. Daoud, T. Qadoummi and D. el Diehn I. Abou-Tair, "An Interactive Rehabilitation Framework for Assisting People with Cerebral Palsy", Proceedings of the 3rd 2015 Workshop on ICTs for improving Patients Rehabilitation Research Techniques - REHAB '15, 2015.

[42] Mullins, J., Mawson, C., \& Nahavandi, S. (2005). Haptic handwriting aid for training and rehabilitation. Systems, Man and Cybernetics, 2005 IEEE International Conference on, 3:2690-2694.

[43] Buljina, A. L., Taljanovic, M. S., Avdic, D. M., \& Hunter, T. B. (2001). Physical and exercise therapy for treatment of the rheumatoid hand. Arthritis Care \& Research, 45:392-397.

[44] Metsis, V., Jangyodsuk, P., Athitsos, V., Iversen, M., \& Makedon, F. (2013). Computer aided rehabilitation for patients with rheumatoid arthritis. Computing, Networking and Communications (ICNC), 2013 International Conference on, pp.97-102. IEEE.

[45] Ryan, R. M., \& Deci, E. L. (2000a). Self-determination theory and the facilitation of intrinsic motivation, social development, and well-being. American psychologist, 55-68.

[46] Ryan, R. M., \& Deci, E. L. (2000b). "Intrinsic and extrinsic motivations: Classic definitions and new directions," Contemporary educational psychology, 25:54-67.

[47] Iversen, M. D., Fossel, A. H., Ayers, K., Palmsten, A., Wang, H. W., \& Daltroy, L. H. (2004). Predictors of exercise behavior at six months in patients with rheumatoid arthritis. Phys Ther, 84:706-716.

[48] Golomb, M.R., Barkat-Masih, M., Rabin, B., Abdelbaky, M., Huber, M., \& Burdea, G. (2009). Eleven Months of home virtual reality telerehabilitation - Lessons learned. Virtual Rehabilitation International Conference 2009, 23-28.

[49] Golomb, M. R., McDonald, B. C., Stuart, S. J., Warden, J., Yonkman, J., Saykin, A., Shirley, B., Huber, M., Rabin, B., AbdelBaky, M., Nwosu, M., Barkat-Masih, M., \& Burdea, G. (2010). In-home virtual reality videogame telerehabilitation in children with hemiplegic cerebral palsy. Arch. Phys. Med. Rehabil., 91:1-18.

[50] Trewin, S. M., Laff, M. R., Cavender, A., \& Hanson, V. L. (2008). Accessibility in virtual worlds. CHI '08 Extended Abstracts on Human Factors in Computing Systems, (Florence, Italy, April 05 - 10, 2008), CHI '08. ACM, New York, NY, 2727-2732.

[51] M. Pirovano, E. Surer, R. Mainetti, P. Lanzi and N. Alberto Borghese, "Exergaming and rehabilitation: A methodology for the design of effective and safe therapeutic exergames", Entertainment Computing, vol. 14 , pp. 55-65, 201

[52] Wilson, G.F., Russell, C. A., \& Davis, I. (2006). The importance of determining individual operator capabilities when applying adaptive aiding. Proceedings of the Human Factors and Ergonomics Society 50th Annual Meeting, pp.141-145. 


\section{HERE TO EDIT) <}

[53] Weiss, P. L., Bialik, P., \& Kizony, R. (2003). Virtual reality provides leisure time opportunities for young adults with physical and intellectual disabilities. Cyberpsychology and Behavior, 6, 335-342.

[54] Wilson, P., Foreman, N., and Stanton, D. (1997). Virtual reality, disability and rehabilitation. Disability Rehab, 19(6), 213-220.

[55] Flores, E., Tobon, G., Cavallaro, F. I., Perry, J. C., \& Keller, T. (2008). Improving patient motivation in game development for motor deficit rehabilitation. Proceedings of the 2008 International Conference on Advances in Computer Entertainment Technology. Yokohama, Japan.

[56] Reinkensmeyer, D. J., \& Housman, S. J. (2007). "If I can't do it once, why do it a hundred times?": Connecting volition to movement success in a virtual environment motivates people to exercise the arm after stroke. Virtual Rehabilitation, 44-48.

[57] Subramanian, S., Knaut, L., Beaudoin, C., McFadyen, B., Feldman, A., \& Levin, M. (2007). Virtual reality environments for post-stroke arm rehabilitation . Journal of NeuroEngineering and Rehabilitation, vol. 4, p. 20.

[58] Ni, T., Bowman, D. A., North, C., \& McMahan, R. P. (2011). Design and evaluation of freehand menu selection interfaces using tilt and pinch gestures. International Journal of Human-Computer Studies, vol. 69, pp. 551-562.

[59] Balaam, M., Rennick Egglestone, S., Hughes, A. M., Nind, T., Wilkinson, A., Harris, E., et al. (2010). Rehabilitation Centred Design. CHI'10 Extended Abstracts on Human Factors in Computing Systems (pp. pp. 4583-4586). ACM.

[60] Gallo, L., Minutolo A., \& De Pietro, G. (2010). A user interface for VRready $3 \mathrm{D}$ medical imaging by off-the-shelf input devices. Computers in Biology and Medicine, 40(3):350-358.

[61] Krabichler, C., Haubner, R., Engelbrecht, M., \& Englmeier, K.H. (1998). VR interaction techniques for medical imaging applications. Computer Methods and Programs in Biomedicine, 56(1):65-74.

[62] https://unity3d.com/

[63] Sivak, M. L. (2012). Dissertation: Design and Implementation of Patient Specific Virtual Reality Systems for Motor Rehabilitation. Boston, MA.: Northeastern University.

[64] Nielsen, J., Landauer, T. K. (1993). "A mathematical model of the finding of usability problems". Proceedings of ACM INTERCHI'93 Conference (Amsterdam, The Netherlands, 24-29 April 1993).

[65] Poels, K., de Kort, Y., \& IJsselsteijn, W. (2007). "It is always a lot of fun!": exploring dimensions of digital game experience using focus group methodology. Proceedings of the 2007 conference on Future Play, Toronto, Canada, ACM, 83-89.

[66] Brooke, J. (1996). SUS: A "Quick And Dirty" Usability Scale. In P. W. Jordan, B. Thomas, B. A. Weerdmeester \& I. L. McClelland, Eds. Usability Evaluation In Industry, pp. 189-194. London: Taylor \& Francis.

[67] Lewis, J. R. (1995). IBM Computer Usability Satisfaction Questionnaires: Psychometric Evaluation and Instructions for Use. International Journal of Human-Computer Interaction, 7:1, 57-78.

[68] Lund A. M. (2001). Measuring usability with the USE questionnaire. Usability and User Experience, vol 8. 